\title{
Research on rapid mapping technology in the field of unmanned aerial vehicle (UAV) aerial survey
}

\author{
Wenjia Du ${ }^{1,2}$, Fengchen Chen ${ }^{1,2, ~}{ }^{*}$, Ke Tang ${ }^{1,2,3}$, Lan Cheng ${ }^{1,2}$, Huanjing Jiao ${ }^{1,2}$ \\ ${ }^{1}$ China Airport Construction Group Co., Ltd., Beijing, China \\ ${ }^{2}$ Beijing Super-Creative Airport Technical Ltd., Beijing, China \\ ${ }^{3}$ School of Automation Science and Electrical Engineering, Beihang University, Beijing, China
}

\begin{abstract}
Through the development of computer image processing technology and UAV technology, after installing high-definition cameras on UAVs and taking high-resolution images, the technology of directly generating DOM images in the surveyed areas using software has been well-developed. However, under the emergency conditions such as dealing with disasters, the long-term processing time required for aerial surveys of UAVs has become a major efficiency constraint to surveying and mapping. This paper focuses on the rapid mapping and mosaicking of survey images from unmanned aerial vehicles under emergency conditions, and elaborates current research status, difficulties and resolvents of the rapid mapping of existing UAVs.
\end{abstract}

\section{Introduction}

In the traditional surveying and mapping process, after measuring the ground object, topographic points, corresponding coordinates and elevation data through the measurement tools such as RTK and total station, it is necessary to process the data in the image processing software. The detailed information of the measured area is reflected by the point, line and surface data. With the development of UAV control technology and image processing algorithms, UAVs are equipped with highdefinition cameras for high-altitude or low-altitude aerial imagery. The collected images and positioning data are unified and processed in digital photogrammetric workstations, which can produce high-definition DOM images and DEM terrain data. Such processes have become mainstream in research and actual mapping.

The process [1] of UAV aerial survey mainly includes:

1) Arrange the ground control point (GCP) in the survey area to obtain the coordinate of the control points for the images;

2) Assign the UAV routes according to the range of the measured area and fly according to the routes;

3) Mark the control points in the images taken by the UAV and use the processing software to operate the aerial triangulation and image mosaicking;

4) Base on requirements, generate DOM, DEM and other mapping results.

After acquiring aerial images from UAV, the image processing mainly includes two parts in the image processing software: tilt correction and image mosaicking.

\section{Tilt correction of UAV image data}

After acquiring the UAV aerial survey data, the traditional way is to use software to rebuild the surveying image based on the aerial images. This section expatiates the principle of tilt correction during the reconstruction process, and describes the problems faced in the reconstruction process with related algorithms and key technologies.

\subsection{Image distortion and correction}

When the camera in UAV captures images, the images' edge is deformed due to the distortion caused by the camera lens itself. This distortion can be named as image distortion. The coordinate distortion of the points on the images can be expressed as [2]:

The main point of the image is $O\left(x_{0}, y_{0}\right)$. The radial distortion coefficient $K_{1}, K_{2}$, and the eccentric distortion parameters are $P_{1}, P_{2} . P\left(x_{i}, y_{i}\right)$ shows the point (image points) position on the distorted image.

$$
\begin{aligned}
& \Delta x=x\left(K_{1} r^{2}+K_{2} r^{4}\right)+P_{1}\left(r^{2}+2 x^{2}\right)+2 P_{2} x y \\
& \Delta y=y\left(K_{1} r^{2}+K_{2} r^{4}\right)+2 P_{1} x y+P_{2}\left(r^{2}+2 y^{2}\right)
\end{aligned}
$$

After determining the camera image distortion mapping relationship, the distorted images are interpolated and resampled to perform distortion correction [3].

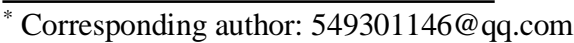




\subsection{Geometric distortion and correction}

Due to the angle tilt of the camera and the angle of the terrain itself, the aerial survey image of the UAV is also deformed. This deformation can be named as geometric distortion.

At present, there are two main conventional methods for geometric distortion correction. One is to use the ground control point (GCP) to generate polynomial fitting correction through aerial triangulation calculation; the other is to use a large scale map to correct by registering with the same name points.

For the tilt correction method based on polynomial fitting, the general polynomial form is like [4]:

$$
\left\{\begin{aligned}
x= & a_{000}+a_{100} X+a_{010} Y+a_{001} Z+ \\
& a_{200} X^{2}+a_{020} Y^{2}+a_{002} Z^{2}+a_{110} X Y+ \\
& a_{101} X Z+a_{011} Y Z+\cdots \\
y= & b_{000}+b_{100} X+b_{010} Y+b_{001} Z+ \\
& b_{200} X^{2}+b_{020} Y^{2}+b_{002} Z^{2}+b_{110} X Y+ \\
& b_{101} X Z+b_{011} Y Z+\cdots
\end{aligned}\right.
$$

$(x, y)$ is the coordinate position for the image point and $(X, Y)$ shows the corresponded ground point for $(x, y)$ with the coefficients $a_{i}$ and $b_{i}$.

Xiao [5] proposed an image correction method using affine invariance. The initial affine matrix of the camera was calculated by estimating the camera axis orientation parameters, and the corrected image was obtained by the inverse affine transformation. $\mathrm{Wu}$ [6] proposed a tilting image correction method based on spatial transformation. The method is based on the image sampling interval to determine the overlapped coordinate domain and coordinate point set of the two neighbouring images, and the corrected coordinates are obtained by using the inverse transformation equations of the model to realize the correction.

\subsection{Geometric distortion correction without control points}

During the actual mapping process, it is very difficult to obtain multiple control points with precise coordinates in the regions of mountains, lakes, oceans, etc. In addition, in uninhabited areas such as deserts and plateaus, it's also hard to get sufficient same-name points in such large-scale maps. Once in the emergency phase which needs fast survey response, there is a lack of time to establish control points. While using UAV mapping, traditional feature point recognition and map registration methods are difficult to perform effectively, and may face the circumstances that processing survey images data without control points, which requires aerial survey research of UAVs without control points recognition.

$\mathrm{Xu}$ [7] proposed a geometric correction method based on POS parameters. In the absence of ground control points, the corrected POS parameters are directly used as image orientation parameters. The image correction is achieved by collinear equations and indirect methods. $\mathrm{Li}$ [8] established a UAV remote sensing image set correction model based on flight attitude change, and proceeded image set correction without control points. $\mathrm{Hu}$ [9] proposed a geometrically correcting method for images using image initial outer orientation elements and digital elevation model data.

\section{Rapid mosaicking and processing of UAV image data}

The traditional aerial survey process mentioned above has the characteristics of long calculation time, and the long processing time seriously reduces the efficiency of surveying and mapping for the post-disaster reconstruction and other mapping scenarios that require rapid mapping. This section analyses the rapid image stitching and presents the current research progress.

As the aerial image of the drone has the characteristics of small displacement and high overlap rate, some characteristics of the image data are used to analyse, calculate and register the same name image point during image mosaicking phase. Several common registration methods [10] include image-based, objectbased, least squares and feature-based image matching. The feature-based image matching, as it adapts to various ground features such as buildings and terrains, and can deal with the situation of discontinuous images and shadow shading well, is suitable for the current UAV aerial image processing.

\subsection{Image stitching based on image features}

The steps [11] of the feature-based image matching strategy are feature extraction, determination of candidate feature points, transformation parameter estimation or final feature correspondence. Among these steps, the image pyramid is generally established before feature extraction, which uses the feature of local invariance to enhance the effect of feature registration. According to the characteristics of aerial imagery, the extracted features generally include point features, line features and surface features. According to different kinds of features, there are different corresponding extraction and matching algorithms and operators.

For the image matching strategy based on point features, the SIFT and SURF descriptors proposed by the local invariance of the image are more commonly used. Based on the feature point invariance of image rotation scaling, these descriptors and algorithms have been widely used as image stitching tools. Also, a lot of improved algorithms for SIFT [12] and SURF [13] have appeared. Gong [14] proposed a way to automatically splicing small-area images using the optimized SIFT feature matching algorithm and using GPS data which are recorded in UAV to assist coordinate processing. Wang [15] proposed a feature point correction splicing method to get SURF features of adjacent images through the POS information in UAV, which also used the gradual adaptive weighting strategy to fuse the images. 


\subsection{Fast stitching optimization of images supported by hardware and video means}

Due to the large number of aerial imagery of UAVs, even with efficient image feature recognition and stitching algorithms, the operation time without control points is very long. Therefore, in addition to improving the stitching speed and stitching accuracy in software algorithms, some scholars have proposed to improve the computer graphics accelerator card GPU technology for processing stitched images. Liu [16] proposed a texture scheduling algorithm combining CUDA technology and Voronoi graph index. It uses NVIDIA's parallel computing architecture based on general GPU computing platform to improve the GPU running speed and improve the image stitching speed.

With the development of real-time image processing technology and UAV products, rapid real-time analysis of video has begun for rapid mapping of aerial surveys of UAVs. During the rapid video processing, surveying video is captured by the UAV during aerial surveying, then the video is quickly compressed in the image processing module of the UAV, and finally transmitted in real time through a mobile network such as $4 \mathrm{G} / 5 \mathrm{G}$. After the processing system receives the video, key frame is extracted among the video stream. Through affine transformation processing of the key frame image, the position of the video key frame is located in this method. And the remaining steps are basically the same as the aerial image processing steps of the UAV. Zhou [17] proposed a method for transforming and splicing the key frames of aerial survey video of UAVs. Sun [18] proposed a set of UAV video processing system, and combined the display of the UAV tracking coordinates in the mapping results. Xue [19] proposed a system that combines UAV video live broadcast and real-time construction of orthophoto model. The current research system for real-time processing of aerial survey video of UAVs is still in its infancy, and has the characteristic that combining multiple usages, such as analysing video information for rapid response of fires [20], which are not completely developed for getting surveying results. However, the real-time processing of the video changes the processing phase of the surveying result from after the outside field work to during the outside field work. In the scene where the surveying result is urgently needed, the speed of mapping can be accelerated, and it can also be regarded as a rapid measurement tools of the UAV images.

\section{Conclusion}

The processes and algorithms for mapping through aerial imagery of UAVs have been carried out to a more advanced stage of research, and several handy software has emerged. However, the algorithm for rapid mapping and hardware development under emergency conditions is still in the early stage, and it is mainly used for the correction and splicing process of aerial image. The processing speed of many software algorithms is still limited by the hardware processing efficiency. With the development of UAVs, software algorithms and hardware technologies, the rapid mapping technology of unmanned aerial photography will be more proficient, and the efficiency of traditional UAV mapping technology will be greatly improved. Through the usages of the rapid mapping technology and UAV surveying technology, the efficiency for the modern constructions such as airports, roads and railways will be highly enhanced with fast-developing and detailed site or scene information models.

\section{Acknowledgements}

This research was funded by the China Civil Aviation Safety Capacity Building Fund Project: Realization and Construction Demonstration of Digital Quality Monitoring System for the Construction of Gravel Pile in Airport Projects.

\section{References}

1. Y. Yang, J. Ji, H. Shi, B. Zhao, Rapid Tilt Photogrammetry Practice Based on Dajiang Consumer UAV, Beijing Surveying and Mapping, 33, 3 (2019)

2. W. Feng, Close-range Photogrammetry: Photographic Determination of Object Shape and Motion State, (Wuhan University Press 2002)

3. Y. Wang, Research on Splicing Algorithm Based on UAV Aerial Image, Master's thesis, Beijing University of Technology, Beijing, China, (2018)

4. J. Duan, L. Lu, H. Wang, Research and Implementation on Geometric Rectification of Areo Images, Journal of Wuhan University of Technology (Transportation Science \& Engineering), 32, 3 (2008)

5. H. Xiao, B. Guo, D. Li, X. Zhao, W. Jiang, H. Hu, C. Zhang, A Quick and Affine Invariance Matching Method for Oblique Images, Acta Geodaetica et Cartographica Sinica, 44, 4 (2015)

6. J. Wu, H. Sun, Efficient Method of Oblique Image Correction, Remote Sensing Technology and Application, 30, 5 (2015)

7. Q. Xu, A Method of Geometric Correction and Mosaic of Unmanned Aerial Vehicle Remote Sensing Image without Ground Control Points, Master's thesis, Nanjing University, Jiangsu, China, (2012)

8. Z. Li, Research on UAV Remote Sensing Geometrical Rectification Technology without Control Points, Master's thesis, University of Electronic Science and Technology of China, Sichuan, China, (2008)

9. H. Hu, Q. Zhu, Z. Du, Y. Zhang, Y. Ding, Reliable Spatial Relationship Constrained Feature Point Matching of Oblique Aerial Images, Photogramm. Eng. Rem. S, 81, 1 (2015) 
10. X. Yu, Z. Lv, D. Hu, Review of Remote Sensing Image Registration Techniques, Optics and Precision Engineering, 21, 11 (2013)

11. P. Wang, Y. Xu, Photogrammetry (Surveying and Mapping Engineering), (Wuhan University Press, Third Edition, 2016)

12. D. G. Lowe, Object Recognition from Local ScaleInvariant Features, ICCV, 99, 2 (1999)

13. H. Bay, A. Ess, T. Tuytelaars, L. V. Gool, SpeededUp Robust Features (SURF), Comput. Vis. Image. Und, 110, 3 (2008)

14. A. Gong, X. He, T. Lei, J. Li, Fast Image Processing Method of UAV without Control Data, Journal of Geo-information Science, 12, 2 (2010)

15. Y. Wang, D. Zhao, N. Zhang, J. Shi, Q. Deng, Stitching Method Based on UAV POS Information, Computer Systems \& Applications, 27, 3 (2018)

16. F. Liu, B. Yang, F. Zhang, S. Xu, Y. Yang, A Fast Visualization Method of UAV Aerial Remotely Sensed Imagery for Emergency Response, Science of Surveying and Mapping, 43, 7 (2018)

17. G. Zhou, Near Real-Time Orthorectification and Mosaic of Small UAV Video Flow for Time-Critical Event Response, IEEE. T. Geosci. Remote, 47, 3 (2009)

18. X. Sun, Y. Li, F. Wang, F. Liu, S. Wang, G. Zhou, Design and Implementation of UAV Geographic Information Video System, Science of Surveying and Mapping, 43, 10 (2018)

19. W. Xue, The Calibration of UAV Video GeoInformation and Live Processing Technology, Master's thesis, PLA Information Engineering University, Zhengzhou, China, (2014)

20. J. Wu, Z. Dong, G. Zhou, Geo-registration and Mosaic of UAV Video for Quick-response to Forest Fire Disaster, MIPPR 2007: Pattern Recognition and Computer Vision, 6788, 678810 (2007) 